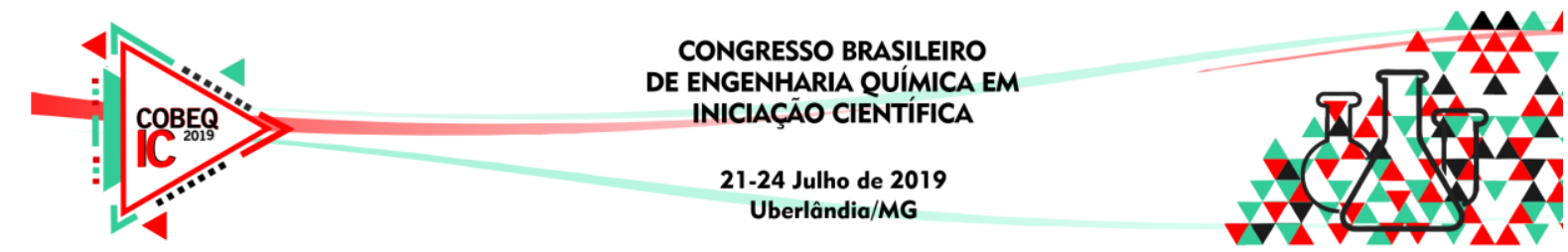

\title{
AVALIAÇÃO DO PROCESSO DE RETICULAÇÃO TÉRMICA SOBRE A INCORPORAÇÃO DE CETOPROFENO EM PARTÍCULAS DE SERICINA E ALGINATO
}

\author{
V. M. S. FREITAS ${ }^{1}$, E. D. FREITAS ${ }^{1}$, P. C. P. ROSA ${ }^{2}$, M. G. C. $\operatorname{SILVA}^{1}$ e M. G. A. \\ VIEIRA $^{1}$ \\ ${ }^{1}$ Universidade Estadual de Campinas, Faculdade de Engenharia Química \\ ${ }^{2}$ Universidade Estadual de Campinas, Faculdade de Ciências Farmacêuticas \\ E-mail para contato: melissagav@ feq.unicamp.br
}

\begin{abstract}
RESUMO - A sericina é uma proteína presente no casulo do bicho da seda e normalmente é um material de descarte da indústria têxtil, porém a sua propriedade de biocompatibilidade e biodegradabilidade desperta o interesse da indústria farmacêutica. Juntamente com o alginato, um biopolímero presente nas algas marrons, a sericina tem a capacidade de formar blendas, com grande aplicabilidade na área farmacêutica. A finalidade do presente trabalho foi avaliar a incorporação do fármaco cetoprofeno em partículas formadas a partir da blenda de sericina e alginato, após passar pelo processo de reticulação térmica. A sericina foi extraída a partir dos casulos do bicho-da-seda Bombyx mori em autoclave, e o alginato foi obtido comercialmente. Para a obtenção das partículas utilizou-se a técnica de gelificação iônica através do método de gotejamento em solução de $\mathrm{CaCl}_{2} 3 \%$. As partículas obtidas foram submetidas ao processo de reticulação térmica em duas etapas subsequentes, a $40{ }^{\circ} \mathrm{C}$ e a $100{ }^{\circ} \mathrm{C}$. Para avaliar as partículas, foram analisados a eficiência de incorporação e o carregamento de fármaco, nos quais se observou que as partículas que passaram pelo processo de reticulação térmica apresentaram resultados melhores, quando comparadas às partículas que não passaram por esse processo.
\end{abstract}

\section{INTRODUÇÃO}

A fibroína é uma proteína insolúvel utilizada na produção dos fios de seda, e está presente em casulos de Bombyx mori, que é popularmente conhecido como bicho-da-seda. Junto a esta proteína, na composição dos casulos, encontra-se a sericina, outra proteína que é comumente descartada pela indústria têxtil. Por ser descartada, a sericina tem sido estudada para diversas aplicações, dentre elas a farmacêutica, especialmente devido à sua capacidade de formar blendas. Uma possível combinação desta formação de blendas é a sua associação com o polímero alginato de sódio (Zhang et al., 2015). O alginato de sódio é extraído de algas marrons e é um polímero biodegradável e biocompatível e, com isso, já é utilizado em algumas áreas da indústria farmacêutica para o encapsulamento de fármacos para que se tornem mais resistentes ao meio gastrointestinal (Lee e Mooney, 2012).

A combinação da sericina com o alginato de sódio para a formação de blendas é útil, pois, por ser gastrorresistente, ela evita que as propriedades do fármaco sejam afetadas pelo 


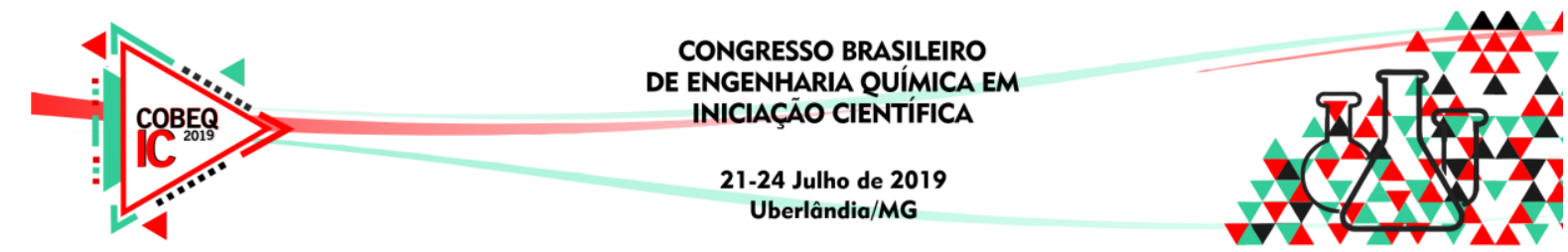

meio gástrico, além de possibilitar uma liberação controlada do mesmo (Vidart et al. 2018). Porém, é possível que o objetivo pretendido não seja atingido somente com a blenda de sericina e alginato, sendo necessária a adição de agentes reticulantes à blenda polimérica. Um exemplo de agente reticulante é a proantocianidina (PA), que é um agente reticulante covalente extraído das sementes de uvas e que, segundo estudos anteriores melhoram a reticulação da blenda de sericina e alginato, podendo colaborar para uma melhor liberação de fármacos com uma meia vida curta (Bezerra, 2018). Além disso, outra técnica que pode ser utilizada visando melhorar ainda mais as propriedades das partículas é a reticulação térmica, que consiste em realizar a secagem das partículas em estufa com temperatura controlada.

Estudos como este, além de garantir uma melhor e prolongada liberação de fármacos no corpo humano, também tem como objetivo reduzir os efeitos colaterais causados pela ingestão de medicamentos. O cetoprofeno é um fármaco com propriedades analgésicas, antiinflamatórias e antitérmicas (Castagnetti e Mariella, 2015), porém sua meia vida é curta (2-3 horas) e quando exposto ao meio gástrico, correntemente causa irritações na mucosa intestinal, sendo por isso um ótimo candidato a este estudo (Kantor, 1986).

Sendo assim, o objetivo do presente trabalho foi a obtenção de partículas de sericina e alginato incorporadas com cetoprofeno, com e sem o agente reticulante PA, submetidas à reticulação térmica a 40 e $100{ }^{\circ} \mathrm{C}$. Para avaliação destas partículas foram analisados a eficiência de incorporação e o carregamento de fármaco.

\section{METODOLOGIA}

\subsection{Material}

Os casulos do bicho-da-seda foram cedidos pela empresa de fiação de seda BRATAC da cidade de Londrina-PR, enquanto o cetoprofeno foi fornecido pela Purifarma e o alginato de sódio, para a formação das blendas, pela Sigma-Aldrich. $O$ agente reticulante proantocianidina foi obtido da empresa Galena Farmacêutica. Para a preparação das soluções utilizou-se fosfato de potássio monobásico e hidróxido de sódio, ambos fornecidos pela empresa Dinâmica, e cloreto de cálcio, pela empresa Anidrol. Todas as soluções foram preparadas utilizando água ultrapura.

\subsection{Parte Experimental}

Extração da sericina: A extração da sericina foi baseada na proposta de Silva (2016), na qual os casulos do bicho-da-seda foram limpos e cortados em pedaços de aproximadamente $1 \mathrm{~cm}^{2}$ de área superficial, lavados com água da torneira e com água ultrapura e logo após secos em estufa a $45{ }^{\circ} \mathrm{C}$. Com os casulos já secos, começou-se efetivamente a extração da sericina, sendo adicionados $40 \mathrm{~g}$ de casulos secos para cada $1 \mathrm{~L}$ de água deionizada em um Erlenmeyer e este, por sua vez, colocado em autoclave à pressão de $1 \mathrm{kgf} / \mathrm{cm}^{2}$ e na temperatura de $120^{\circ} \mathrm{C}$, durante $40 \mathrm{~min}$. Para completar a etapa de extração, foi necessário filtrar a solução obtida, ficando a fibroína retida no papel filtro e a sericina separada no filtrado. A solução foi reservada em um recipiente fechado à temperatura ambiente para sua estabilização. 


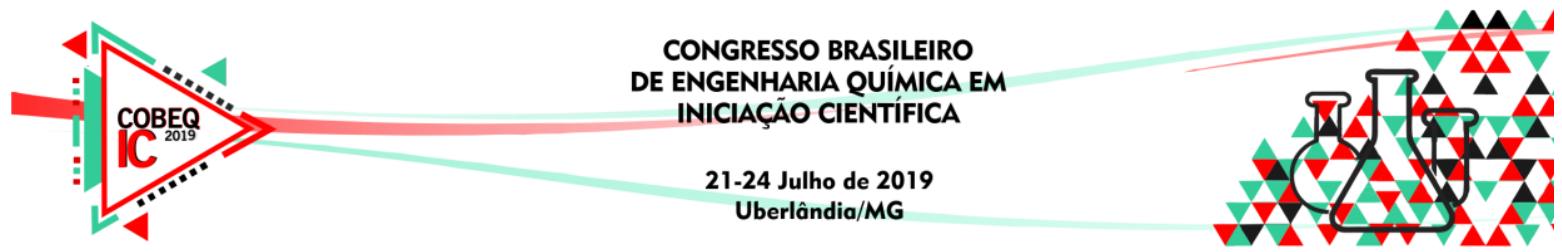

Fracionamento da sericina: Depois de estabilizada, a solução de sericina foi levada ao congelador por $24 \mathrm{~h}$ e, depois deste período, foi descongelada à temperatura ambiente para que ocorresse a separação em duas frações, como proposto por Silva (2014). Uma das frações obtidas é aquosa e a outra é insolúvel em água e de maior massa molecular, sendo esta a utilizada na preparação das blendas. Logo após a separação foi realizado o método das massas visando ajustar a concentração da solução de sericina para 2,5\% (m/V) (Silva et al., 2013).

Desenvolvimento da blenda: Para a preparação da blenda de sericina e alginato foi realizado método similar ao proposto por Vidart et al. (2018). Para isso, a solução de sericina com a concentração já ajustada passou por um processo de re-aquecimento em autoclave a $120{ }^{\circ} \mathrm{C}$ e à pressão de $1 \mathrm{kgf} / \mathrm{cm}^{2}$, por $10 \mathrm{~min}$. Em seguida a solução foi agitada em um Ultraturrax ${ }^{\circledR}$ a $4000 \mathrm{rpm}$ até que sua temperatura atingisse $55^{\circ} \mathrm{C}$, para que então o alginato fosse adicionado, mantendo sob agitação até sua completa dissolução. Atingindo-se a homogeneidade da solução, o cetoprofeno foi adicionado e agitado inicialmente a $4000 \mathrm{rpm}$ e ao final, a $8000 \mathrm{rpm}$, para que o fármaco fosse completamente dissolvido. No caso da formulação reticulada covalentemente, a PA foi adicionada após esta etapa e agitada de forma similar ao fármaco, até a homogeneização. Cada formulação desenvolvida está descrita na Tabela 1, sendo que cada composição foi baseada nos melhores resultados anteriores obtidos pelo grupo de pesquisa (Freitas et al., 2018; Freitas et al., 2019), sem a etapa de reticulação térmica.

Tabela 1 - Composição das formulações obtidas no presente trabalho. Todas as concentrações são dadas em \% (m/V)

\begin{tabular}{|c|c|c|c|c|}
\hline Formulação & $\begin{array}{c}\text { Sericina } \\
\%(\mathrm{~m} / \mathrm{V})\end{array}$ & $\begin{array}{c}\text { Alginato } \\
\%(\mathrm{~m} / \mathrm{V})\end{array}$ & $\begin{array}{c}\text { Cetoprofeno } \\
\%(\mathrm{~m} / \mathrm{V})\end{array}$ & $\begin{array}{c}\text { Proantocianidina } \\
\%(\mathrm{~m} / \mathrm{V})\end{array}$ \\
\hline \hline TCSA & 2,5 & 2,0 & 4,0 & - \\
\hline TCPA & 2,5 & 2,0 & 4,0 & 0,5 \\
\hline
\end{tabular}

Preparação das partículas: Para a obtenção das partículas, as soluções contendo o fármaco passaram pelo processo de gelificação iônica (Vidart et al., 2018), no qual a solução foi gotejada em solução de cloreto de cálcio $\left(\mathrm{CaCl}_{2}\right) 3 \%$ sob agitação magnética, para que a gelificação ocorresse de forma instantânea. Após esta etapa as partículas foram colocadas sob agitação em jar test a 100 rpm durante 30 min, com o objetivo de completar a reticulação. Por fim, as partículas foram submetidas à secagem em estufa a $40{ }^{\circ} \mathrm{C}$, seguida de outra etapa a $100^{\circ} \mathrm{C}$, durante 24 horas cada uma, sendo que esses valores foram aplicados conforme reportado como melhores resultados em trabalhos anteriores (Silva, 2016).

Avaliação da Eficiência de incorporação: Para que fossem realizadas as avaliações da Eficiência de incorporação para as formulações da Tabela 1, foi utilizado um método adaptado de Sinha et al. (2015). Sendo assim, 0,1 g de partícula foram colocados em contato com $500 \mathrm{ml}$ de solução tampão fosfato $(\mathrm{pH} \mathrm{6,8)} \mathrm{e} \mathrm{mantidas} \mathrm{em} \mathrm{repouso} \mathrm{por} 24 \mathrm{~h}$. Após esse período a solução foi sonicada durante $15 \mathrm{~min}$, a fim de que todo o cetoprofeno presente nas formulações fosse liberado. A solução foi então filtrada em filtro $0,45 \mu \mathrm{m}$ e a concentração de fármaco foi obtida por espectrofotometria UV-visível no comprimento de onda de $258 \mathrm{~nm}$. A Eficiência foi calculada conforme apresentado na Equação 1. 


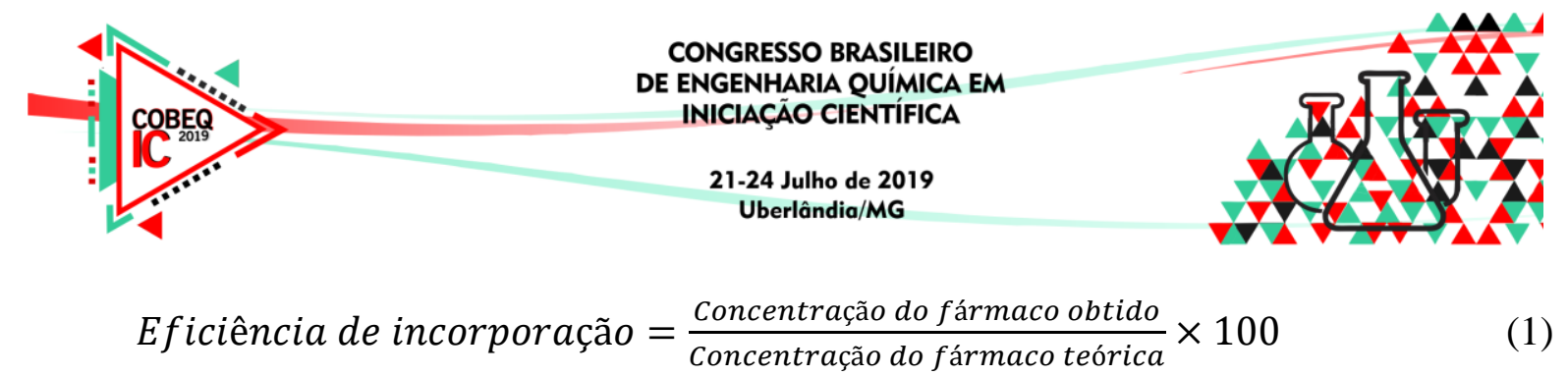

Sendo a Concentração do fármaco obtido o resultado obtido da espectrofotometria UV-visível e a Concentração do fármaco teórica, a quantidade inicial adicionada de fármaco.

Avaliação do carregamento de fármaco: A metodologia para a determinação do carregamento de fármaco foi a mesma utilizada para a avaliação da Eficiência de incorporação, sendo o Carregamento dado pela Equação 2.

$$
\text { Carregamento }=\frac{\text { Massa de fármaco na partícula }}{\text { Massa da partícula }} \times 100
$$

\section{RESULTADOS E DISCUSSÃO}

\subsection{Avaliação da Eficiência de Incorporação}

A Figura 1 apresenta os resultados obtidos para as eficiências de incorporação das formulações presentes na Tabela 1. Para efeitos de comparação, também são apresentados resultados reportados anteriormente para as mesmas formulações, sem a etapa de reticulação térmica (CSA - sericina/alginato/cetoprofeno e CPA - sericina/alginato/cetoprofeno/ proantocianidina), obtidos pelo grupo de pesquisa (Freitas et al., 2018; Freitas et al., 2019).

Figura 1 - Eficiências de incorporação das formulações TCSA, TCPA, CSA e CPA.

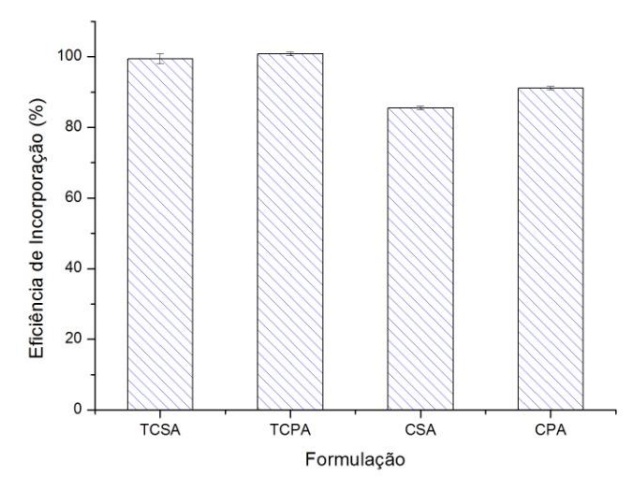

Pode-se observar da Figura 1 que as partículas TCSA e TCPA, que passaram pelo processo de reticulação térmica, apresentaram uma eficiência maior quando comparadas às partículas que foram secas à temperatura ambiente. Portanto conclui-se que a reticulação térmica contribuiu para o aumento da incorporação do fármaco. Isso ocorre devido às mudanças que o aquecimento causa na estrutura da proteína, como a formação de ligações dissulfeto, melhorando a reticulação da partícula e, portanto, o aprisionamento do fármaco.

Nota-se também que as partículas que passaram pela reticulação térmica atingiram valores bem próximos a $100 \%$, podendo até ultrapassar esse valor, considerando-se a barra de erros. Isso pode ter ocorrido porque no cálculo da eficiência considera-se a concentração de fármaco teórica nas partículas. Assim, qualquer perda de matéria-prima durante a produção das partículas influencia diretamente nesse parâmetro. 


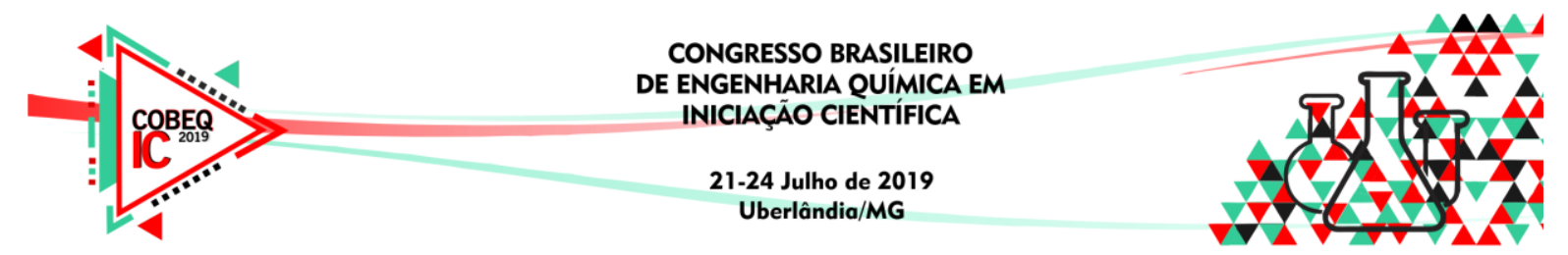

\subsection{Avaliação do Carregamento}

Para melhor avaliar as características das formulações apresentadas na Tabela 1, também se avaliou o carregamento de fármaco das mesmas a partir da Equação 2. Os resultados estão demonstrados na Figura 2.

Figura 2 - Valores de carregamento de fármaco para as formulações TCSA, TCPA, CSA e CPA.

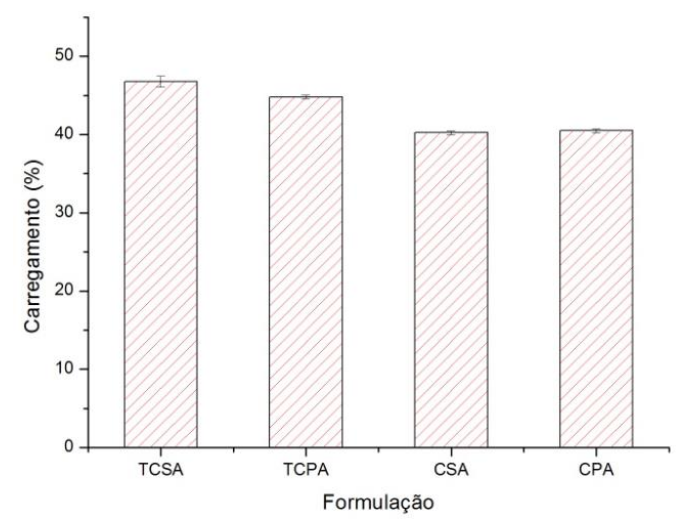

O carregamento expressa a quantidade de fármaco presente na partícula em relação à massa das partículas, portanto quanto maior o seu valor, menor será a quantidade de partículas requeridas para atingir um mesmo efeito terapêutico. Pode-se observar que o carregamento para as partículas que sofreram reticulação térmica tem valores entre $45 \%$ e $48 \%$, sendo que nas partículas que secaram a temperatura ambiente esses valores são de aproximadamente $40 \%$, tanto para as formulações com a presença de proantocianidina quanto as apenas com sericina, alginato e fármaco. Portanto, assim como no caso da eficiência de incorporação, a reticulação térmica também foi capaz de aumentar o carregamento, devido à sua capacidade de favorecer a reticulação das partículas.

\section{CONCLUSÕES}

Com os resultados apresentados, pode-se concluir que as partículas submetidas à reticulação térmica a 40 e $100{ }^{\circ} \mathrm{C}$ apresentaram resultados de eficiência de incorporação e carregamento satisfatórios e melhores, quando comparados às partículas que não passaram pelo mesmo processo. Tal fato está associado à alteração na estrutura das proteínas, ao passar pelo aumento de temperatura, melhorando sua reticulação.

As formulações com o agente reticulante covalente, a proantocianidina, apresentaram uma melhor eficiência de incorporação do que as formulações em que ele não está presente, demonstrando sua importância para esse parâmetro. Porém, quando se observa o carregamento do fármaco, as partículas sem reticulação térmica (CSA e CPA) apresentaram valores muito próximos, enquanto no caso das que sofreram reticulação térmica, na presença do agente reticulante covalente, observa-se um menor carregamento em relação à partícula formada apenas por sericina, alginato e cetoprofeno.

\section{AGRADECIMENTOS}




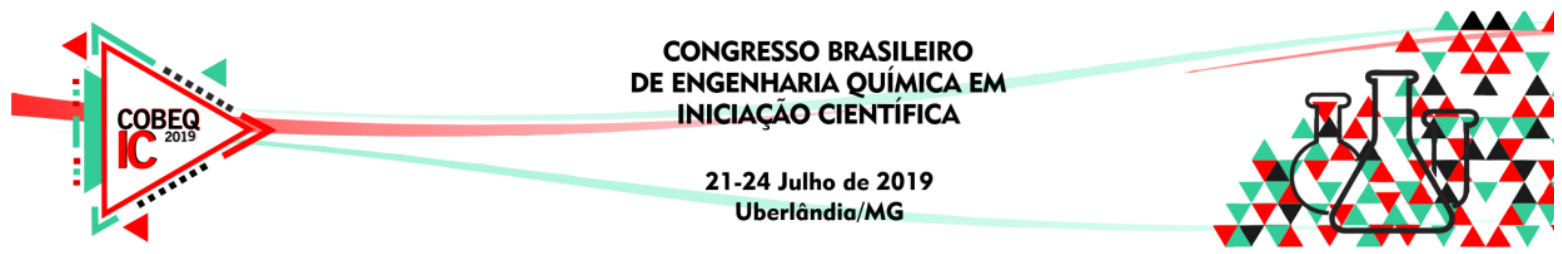

Os autores agradecem o apoio financeiro recebido do CNPq (Proc. 301401/2016-0), CAPES e FAPESP (Proc.2015/13505-9) e à empresa BRATAC pelo fornecimento dos casulos.

\section{REFERENCIAS}

BEZERRA, I. C. S. Incorporação de furosemida em matriz gastrorresistente de sericina e alginato para liberação modificada. FEQ/UNICAMP, Campinas - SP, 92p., 2018. (Dissertação de mestrado).

CASTTAGNETTI, C.; MARIELLA, J. Anti-inflammatory Drugs in Equine Neonatal Medicine. Part I: Nonsteroidal Anti-inflammatory Drugs. J. Equine Vet. Sci., v. 35, p. 475-480, 2015.

FREITAS, E. D., LIMA, B. M., ROSA, P. C. P., SILVA, M. G. C., VIEIRA, M. G. A. Evaluation of proanthocyanidin-crosslinked sericin/alginate blend for ketoprofen extended release. Adv. Powder Technol., 2019. In press.

FREITAS, E. D., ROSA, P. C. P., SILVA, M. G. C., VIEIRA, M. G. A. Development of sericin/alginate beads of ketoprofen using experimental design: Formulation and in vitro dissolution evaluation. Powder Technol., v. 335, p. 315-326, 2018.

KANTOR, T. G., Ketoprofen: a review of its pharmacologic and clinical properties, Pharmacoterapy, v. 6, p. 93-102, 1986.

LEE, K. Y., MOONEY, D. J. Alginate: properties and biomedical applications. Prog. Polym. Sci., v. 37, p.106-126, 2012.

SILVA, T. L. Desenvolvimento e avaliação de partículas à base de blendas entre sericina e alginato para aplicação ambiental. FEQ/UNICAMP, Campinas - SP, 192p, 2016. (Tese de doutorado).

SILVA, T. L., SILVA JUNIOR, A. C., RIBANI, M., VIEIRA, M. G. A., GIMENES, M. L., SILVA, M. G. C. Evaluation of molecular weight distribution of sericin in solutions concentrated via precipitation by ethanol and precipitation by freezing/thawing, Chem. Eng. Trans., v. 38, p.103-108, 2014.

SINHA, P., UBAIDULLA, U., HASNAIN, M. S., NAYAK, A. K., RAMA, B. Alginate-okra gum blend beads of diclofenac sodium from aqueous template using $\mathrm{ZnSO}_{4}$ as a crosslinker. Int. J. Biol. Macromol., v. 79, p. 555-563, 2015.

VIDART, J. M. M., SILVA, T. L., ROSA, P. C. P, VIEIRA, M. G. A., SILVA, M. G. C. Development of sericin/alginate particles by ionic gelation technique for the controlled release of diclofenac sodium. J. Appl. Polym. Sci., v. 135, p. 45919, 2018.

ZHANG, Y., LIU, J., HUANG, L., WANG, Z., WANG, L. Design and performance of a sericin-alginate interpenetrating network hydrogel for cell and drug delivery. Sci. Rep., v. 5 , p. $1-13,2015$. 